Review Article

\title{
Mechanism and Classification of Coal and Gas Outbursts in China
}

\author{
Qi Zhang $\mathbb{D}^{1},{ }^{1}$ Chun-li Yang, ${ }^{2}$ Xiang-chun Li $\mathbb{D}^{1},{ }^{1}$ Zhong-bei $\mathrm{Li}^{1}{ }^{1}$ and $\mathrm{Yi} \mathrm{Li}^{1}$ \\ ${ }^{1}$ School of Emergency Management and Safety Engineering, China University of Mining and Technology-Beijing, \\ Beijing 100083, China \\ ${ }^{2}$ Occupational Hazards Control Technology Center, Beijing Municipal Institute of Labour Protection, Beijing 100054, China
}

Correspondence should be addressed to Xiang-chun Li; chinalixc123@163.com

Received 1 March 2021; Revised 9 April 2021; Accepted 28 April 2021; Published 13 May 2021

Academic Editor: Jia Lin

Copyright (c) 2021 Qi Zhang et al. This is an open access article distributed under the Creative Commons Attribution License, which permits unrestricted use, distribution, and reproduction in any medium, provided the original work is properly cited.

\begin{abstract}
Coal and gas outburst is a kind of complex dynamic disaster with short duration and strong explosiveness, and the modes and strength of the outburst are determined by the in situ stress, gas pressure, and physical and mechanical properties of the coal mass. In this paper, the status quo of research on the mechanism of coal and gas outburst in China is described from three aspects: the controlling effect of single factor, the controlling effect of multi-factor, and new understandings of the outburst mechanism in recent years. Firstly, controlling factors of coal and gas outburst are classified for an in-depth analysis of the main factors of the same type of disasters, and the research progress and new understandings of the mechanism of coal and gas outburst are systematically sorted out. Secondly, the influencing factors of the strength coal mass are analyzed, and the related issues of coal mass strength on coal and gas outburst disaster mechanism are discussed. The results show that the stages of incubation, occurrence, development, and stop on coal and gas outburst are affected by the coupling effects of in situ stress field, gas pressure field, and seepage field, and the coal strength becomes an important factor affecting outburst strength under the same in situ stress and gas pressure. Therefore, the scientific and reasonable improvement methods of such similar simulation experiment devices are proposed according to the existing experimental methods and devices, which is of great significance to provide ideas for the continuous transferring to deep mining and preventing coal and gas outburst in China in the future.
\end{abstract}

\section{Introduction}

Coal and gas outburst is a lump of typical coal and rock dynamic disaster in the process of coal mine production, which has become a key problem affecting the safety of coal production and causing serious casualties and property losses $[1,2]$. Besides, coal and gas outbursts are extremely complex dynamic disasters with extremely short duration and strong explosiveness, and its disaster mechanism is fundamental to prevent coal and gas outburst $[3,4]$.

Currently, the mechanisms of coal and gas outburst are still in the stage of hypothesis, such as the hypothesis of gas action, ground stress, chemical action, and other single factor hypothesis, which cannot fully explain some special disasters reasonably [5-7]. However, in the practical explorations of the mechanism of coal and gas outburst, more and more scholars believe that coal and gas outbursts occur under the combined action of three main factors, namely, gas pressure, in situ stress, and physical and mechanical properties of coal mass $[8,9]$. Since the 1950 s, the former Soviet Union expert B. B. Hodot conducted the simulation experiments on coal and gas outburst with soft coal for the first time, subsequently followed by a lot of coal and gas outbursts by many other researchers, but most of them utilized pulverized coal as the experimental materials and focused mainly on soft coal outburst. Besides, the coal strength is one of the important parameters of the coal physical and mechanical properties of coal, but it was not taken as the variable index of outbursts [10-12]. It is found that coal strength is closely related to outburst indexes such as coal quality, outburst distance, and hole depth. As a result, the change of coal strength affects the outburst strength under the same in situ stress and gas pressure [13-15].

Given this, the paper expounds the disaster mechanisms of coal and gas outbursts in China, assorts the types of the disaster mechanisms, and analyzes the main controlling 
factors of coal and gas outburst. Besides, the influencing factors of coal strength and the causes of coal outburst are emphatically analyzed. Furthermore, the relevant scientific problems are discussed, and the research directions and urgent scientific problems about the mechanism of coal and gas outburst are put forward, so as to provide theoretical support for the prevention and control of coal and gas outburst accidents and the reduction of casualties and property losses.

\section{Research Progress on Mechanism of Coal and Gas Outburst}

The outburst processes occur with huge energy effects and sounds, which damage the facilities in the surrounding rock roadway facilities and discharge with harmful gases such as gas and carbon dioxide, resulting in suffocation casualties, which easily lead to gas explosion, fire, and other safety accidents [16-20]. Since the first recorded coal and gas outburst accident in 1834, it has become one of the most serious disasters in coal mines [21].

Since the 20th century, a large body of researches on the mechanism of coal and gas outbursts, and based on the experience of preventing and controlling outburst accidents [22-24]. At the same time, with laboratory tests and computer simulations, a series of hypotheses on the disaster mechanism are formed, such as spherical shell instability hypothesis [25-27], rheological hypothesis [28, 29], twophase fluid hypothesis [30], unified instability theory [31], fluid-solid coupling instability theory [32, 33], and key layer stress wall outburst mechanism $[34,35]$. In recent years, with the progress of technology and experimental methods, new theories and understandings are constantly put forward, which are mainly summarized as the following aspects.

2.1. Single-Factor Dominant Control Theory. The in situ stress, gas pressure, and the physical and mechanical properties of coal and rock mass fully determine the way and intensity of coal and gas outbursts. According to the current complex geological conditions and the control factors of the outburst, coal and gas outburst disasters can be classified with quantitative study for an in-depth exploration of the main control factors under the same type of coal and gas outburst disasters. Yuan [36] conducted experiments based on the comprehensive action hypothesis and found that coal and gas outbursts were mainly driven by gas. A large body of gas is stored in fractured coal, which supports the formation and growth of the bursts. Driven by the gas, the coal mass of low permeability are destroyed instantly, discharging huge energy and leading to coal and gas outbursts. Chen et al. [37] utilized the coal and gas outburst test system with multi-field coupling conditions and found that gas pressure had obvious pulverization effect on coal mass. The high gas pressure gradient of the gas makes it circulate in the cracks inside the coal mass, and a large amount of pulverization, particles, and small pieces of coal ash inhibit the further outburst.

Moreover, increasing studies show that coal and gas outbursts are closely related to geological tectonic movement, and the uneven distribution of geological structure greatly changes the state of other factors such as in situ stress, gas pressure, and physical and mechanical properties and controls the process of coal and gas outburst. Cheng et al. and He and Chen [38, 39] found that tectonic stress controls the migration and occurrence of gas in coal seam by using the combination of theoretical analysis and field examples, which is the premise and basis of high gas stress in the coal mass, and is also the main controlling factor of coal and gas outburst accidents. Zhang and Liu et al. $[40,41]$ believed that the geological tectonic movement changed the closing-expansion states of the internal microstructures of the coal and rock mass, which promotes the development of the tectonic coal and the occurrence state of gas in the coal and rock mass, and controlled the occurrence of coal and gas outburst accidents. Han and Zhang [42] analyzed that the relationship between coal and gas outbursts and the tectonic evolution in North China, Northeast, and South China with tectonic evolution as the timeline. And they also found that large tectonic areas in the same region would evolve different small-level tectonic structures, which plays a controlling role in these small-scale tectonic regions. Besides, the factors such as coal structure, gas, and in situ stress in different regions have different impacts on each stage of coal and gas outbursts. Yan et al. [43] investigated and summarized the research on coal and gas outbursts in China and abroad and found that the geological conditions in high gas concentration are the prerequisite and key factors for the formation of outbursts. Besides, the destruction and permeability of the coal seam are changed due to the progress of mining and other works, resulting in the desorption of a large number of gases in the coal seam. As a result, the gas pressure increases sharply, and coal and gas outburst accidents occur.

In addition, it is also found that coal and gas outburst disasters are caused by the evolution of paleotectonic stress fields and modern stress fields. Zhu and $\mathrm{Xu}$ [44] found that the tectonic stress fields are the link connecting the in situ stress, gas pressure, and coal physical and mechanical properties, as an indivisible whole [44]. The long-lasting stable states of paleotectonic stress fields are the main reason for the intact existence and high concentration of the gas, while the continuous changes of modern tectonic stress fields are the main reason for the formation of high in situ stress and high gas pressure. In short, the evolution of the tectonic stress fields has become the main controlling factor for coal and gas outbursts.

What is more, the distribution of in situ stress and the weak geological structures in coal seam also play a role in controlling role in coal and gas outbursts. Shu et al. [45] believed that the primary coal mass transforms into structural coal mass due to the change of in situ stress and forms closed high gas stress environments, which accelerates the crushing process of tectonic coal and provides a good material and energy basis for the outburst excitation process. Meanwhile, the key structure model of coal and gas outburst is shown in Figure 1. The key structure 1 with the outburst hazard is the energy source and prerequisite for coal and gas outbursts, and the supporting structure 2 of the key structure 




Figure 1: The key structure model of coal and gas outburst [45].

1 in the coal mass makes its gas and energy accumulate. At the same time, the supporting structure 3 and supporting structure 4 of the roof-floor of coal seam store a large amount of elastic potential energy, which promotes the continuous derivation and expansion of supporting structure 2. Eventually, the accumulated gas and elastic potential energy are released, resulting in coal and gas outbursts.

In Jiangjunling and Malingshan coal mine [46, 47], the sliding structures change the occurrence environments of stress, gas, and physical and mechanical parameters in coal seam, and they also found that the gas content gradient of the coal mass affected by the sliding structures is more obvious than that of the coal mass not affected by them. Furthermore, the reverse fault structures formed by the trailing edge of the sliding structures have better sealing; thus, the gas cannot spread easily and the gas pressure is high, more likely to cause outburst accidents. At present, computer technology has become an important means to understand the mechanism of coal and gas outbursts. Zhang et al. [48] conducted a series of numerical simulation experiments by using $\mathrm{RFPA}^{2 \mathrm{D}}$-Flow solid-gas coupling dynamics software and analyzed the disaster mechanism of coal and gas outbursts in the process of cross-entries from the meso-perspective. In this case, the combined action of high gas pressure gradient, self-weight stress, stress concentration, and blasting stress shock is the main reason for the occurrence of coal and gas outbursts. And the stress concentration in front of tunneling plays an important role in the process of coal and gas outbursts, so that reduces stress concentration in front of tunneling work is an important measure and means to effectively reduce the coal and gas outburst.

Thus, single factors such as gas, in situ stress, and tectonic movement control the occurrence of coal and gas outburst accidents, but the single factor dominant control theory is based on specific geological conditions. The main controlling factors lead to coal and gas outburst by controlling other key factors and can theoretically explain the mechanism of coal and gas outbursts in some mines. Over the years, it is found that the processes of coal and gas outbursts have experienced four stages: preparation, stimulation, development, and stop $[49,50]$, which is inevitably accompanied with changes of in situ stress, gas, and physical and mechanical properties of the coal mass. However, it is difficult to explain the process and critical conditions of the four stages of outbursts. Also, in situ stress, coal rock, gas, and other necessary conditions of coal and gas outbursts should be regarded as an organic system, and the coupling effects of various elements in each stage jointly lead to the coal and gas outbursts $[51,52]$.

\subsection{Disaster-Causing Mechanism of Multi-Field Coupling.}

Coal and gas outbursts are closely connected with the changes and coupling effects of in situ stress field, electromagnetic field, seepage field, and gas pressure field. Under the multi-field coupling conditions, the mechanical properties of gas-bearing coal and the occurrence state of gas are changed, which accelerates the instability of gas-bearing coal and rock and further leads to coal and gas outbursts. For example, the physical model of blasting disturbance structure of coal and rock outbursts was proposed in 2014. The model believes that the existence of the blasting stress wave in the outburst preparation stage breaks the equilibrium state of the initial stress [53], which makes the coal crack develop and forms the "geological weak face." Also, the coupling effect of the gas pressure field and the stress field accelerates the crack propagation, which lead to a large number of gas desorption under the adsorption state. Besides, the coupling effect of gas pressure and blasting stress wave exacerbates coal and rock mass and accelerates the instability of "geological weak face." Eventually, when the gas pressure in the coal seam reaches the critical state, the internal energy and elastic energy of the gas will burst out of the broken coal, leading to the occurrence of coal and rock outbursts.

The coal mining industry has shifted to the deep mining stage, and coal seam mining conditions are more complicated, which leads to the occurrence of coal and rock outbursts frequently. And the gas-bearing coal mass from the deep mining is in a three-dimensional complex in situ stress field [54]. Coal crushing in the mining face is caused 
by the combined action of in situ stress and gas pressure, and the in situ stress becomes an important external factor to induce outbursts. Besides, gas pressure is the key internal power source for outburst initiation, and the experiments also prove this conclusion.

Moreover, the occurrence of coal and rock dynamic disasters involves many theories, such as the adsorption desorption, gas dynamics, seepage, and gas-solid two-phase flow theory. Sun et al. [55], by using classical gas dynamics theory, defined the two-phase flow, in the outburst cavity, reaching the sound velocity as a critical state, and the twophase flow below the critical state was defined as nonexpansion state. Besides, the instantaneous expansion of gas in lots of the nonexpansion two-phase flow will produce a huge momentum effect and burst out disasters in the outburst cavity. Xie et al. [51] also believed that the gas pressure provides the key power source for the initiation of outbursts. Under the coupling effect of in situ stress and gas leads to the continuous derivation and expansion of cracks, pores, and other defect structures in the coal mass, and they also extend along the direction of maximum tangential stress. Furthermore, when the energy of the gas power source is much greater than the energy consumed by the development and penetration of the cracks, the coal mass will collapse and breaks all of a sudden, which will form the coal and gas outburst accidents. Besides, Zhao et al. [56] conducted a series of experiments and found that there are obvious multi-physical field coupling effects between temperature and gas pressure field. After the outburst occurs, a large amount of gas is discharged, which causing the medium gas pressure to reduce. At the same time, the temperature is obviously reduced due to the endothermic reaction during the desorption.

With the development of computer and numerical technology, scholars begin to consider the coupling effect of damage field and fracture field, Zhao et al. [57] carried out many numerical simulation experiments and established the multi-field coupling model of gas pressure field, in situ stress field, damage field, and seepage field. They found that the mining disturbance causes the fracture closure and permeability reduction in the stress concentration area in front of the working face. The stress distribution in front of mining working face is shown in Figure 2. They also found that the mining disturbance would cause closure of the fracture and reduction of permeability in the area of stress concentration in front of the working area, thus increasing the gas pressure gradient in the area of stress relief and the area of stress concentration, which affects the occurrence of coal and gas outburst. Similarly, Zhang et al. [58] came to a similar conclusion that the coal and rock mass gradually break under the coupling of the mining stress field and fracture field, which leads to an increased gas concentration and pressure; driven by the gas pressure, the coal and rock outbursts occur. Nevertheless, Li et al. [59] found that the coupling effect of the vibration field, gas pressure field, in situ stress field, electromagnetic field, and seepage field affects the occurrence of coal and gas outbursts. The vibration caused by blasting and other excavation works further promotes the coupling effect between each other.
Through combining and analyzing related documents on the disaster mechanism of coal and gas outbursts under the coupling effect of multiple fields $[16,60]$, the in situ stress, gas pressure, and coal physical and mechanical properties have obvious differences in the various stages of coal and rock outbursts. And other internal and external factors, such as tectonic movement and mining disturbance, have changed the stress field environments of coal and rock, which accelerated the expansion and damage of coal and rock fractures. In this stage, it mainly depends on the crushing ability of in situ stress of mining stress to coal and rock, which provides the possibility for the occurrence of outbursts. Meanwhile, the adsorption and desorption state of gas changes due to the coupling effect of the in situ stress field and mining stress field, which changes the closing and open states of fractures and increases the internal energy of gas stored in coal and rock. When the accumulated gas internal energy and elastic energy makes the coal and rock mass broken to a certain extent and exceed the energy consumed by coal and rock breaking, which will cause the gas internal energy and elastic energy bursting out rapidly.

2.3. New Understanding of the Mechanism of Coal and Gas Outburst. In recent years, some new theories have been obtained by some advanced techniques on coal and rock outbursts, which can explain some special phenomena of coal and gas outburst. Guo et al. [61] found that there was an obvious stick-slip instability phenomenon during the outburst processes by referring to the mechanism of stick-slip instability in rock bursts [53]. And they also proposed the mechanism of stick-slip instability in coal and gas outbursts, which can reveal the mechanism of vibration fluctuation, delayed outbursts, and intermittent outbursts during the outburst. Besides, Wang et al. [62] constructed the adsorption model between coal particle surfaces and methane gas by using the quantum chemical density functional theory (DFT) calculation method from a view of microscopic. They also found that the electromagnetic waves in the process of deformation and the failure of surrounding coal and rock in working face are due to the vibration, mining, and other works. Furthermore, coal and $\mathrm{CH}_{4}$ molecules are adsorbed to form a companion molecular system that absorbs electromagnetic waves in a quantized form, which leads to $\mathrm{CH}_{4}$ transforming from adsorption state to free state. Finally, a greater gas pressure is formed, which leads to $\mathrm{CH}_{4}$ breaking through the structural surfaces and coal and gas outbursts. $\mathrm{Ma}$ and $\mathrm{Yu}$ [63] regarded the outburst coal mass as the pressure-bearing loose body, and they calculated the motion catastrophe potential function in the process of coal and gas outburst combined with the catastrophe theory from the perspective of the momentum of the coal-bearing loose body. Besides, they also analyzed the influencing factors and disaster-causing mechanism of the coal and gas outburst, which further put forward the mechanism of uncontrolled outburst of pressure-bearing loose body in coal and gas outburst.

Nowadays, Ma et al. [64-67] put forward the hypothesis of butterfly coal and gas outbursts in tunneling roadways 


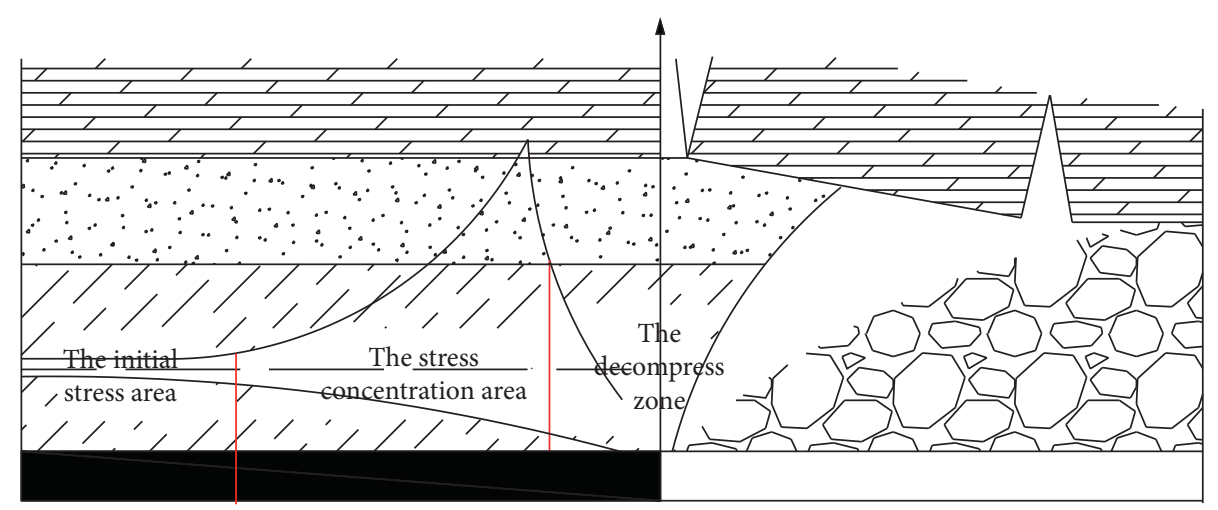

FIgURE 2: The stress distribution in front of mining working face [57].

based on the butterfly rock burst theory and nonlinear dynamic system theory, and they also established the deformation and failure model of circular roadway in the twoway nonisostatic pressure stress field. The theoretical calculation of butterfly-shaped plastic zone is shown in Figure 3 . When the ratio of the maximum principal stress to the minimum principal stress increases continuously, the surrounding rock around the roadway will produce a plastic zone similar to the "Butterfly" shape. Besides, based on the Mohr-Coulomb failure criterion, the implicit equation of the plastic zone boundary of circular roadway surrounding rock in nonisobaric stress field is derived, which is called butterfly failure theory. Moreover, the conjecture holds that a certain amount of butterfly plastic zones appearing in the heading face in a short time due to the uneven distribution of in situ stress. When the increments of the plastic zones increase to the critical value, the elastic potential energy and gas energy stored in the coal rock mass will be released instantly, which thus leading to the coal and gas outburst.

It is believed that the hypothesis of stick-slip instability mechanism and microscopic outburst mechanism lead to a new idea and direction for the study of the mechanism of coal and gas outburst, which can explain specific coal and gas outbursts under certain conditions. However, it fails to give a judgement standard of the risk of coal and gas outbursts and is insufficient to a universal application to the outburst accidents, nor in applications to the coal seam with complicated geological conditions and burial conditions. Besides, as per the analysis, the outburst mechanism of the uncontrolled pressure-bearing loose mass holds that the pressure-bearing loose mass, within the range from the stress concentration area to the boundary of the pressure relief zone in front of the working face, is a practical engineering problem and in line with the characteristics of the general outburst coal mass. But the catastrophe potential function of the motion of the pressure-bearing loose mass, by the hypothesis, operates in the condition that the stress in front of the working face is in circular distribution and the coal mass in the stress concentration area is isotropous. And the original in situ stress and gas pressure are isobaric in all directions, it is different from the actual complex in situ stress environments of coal and gas outbursts, and there is a certain error with the actual movement increment about the

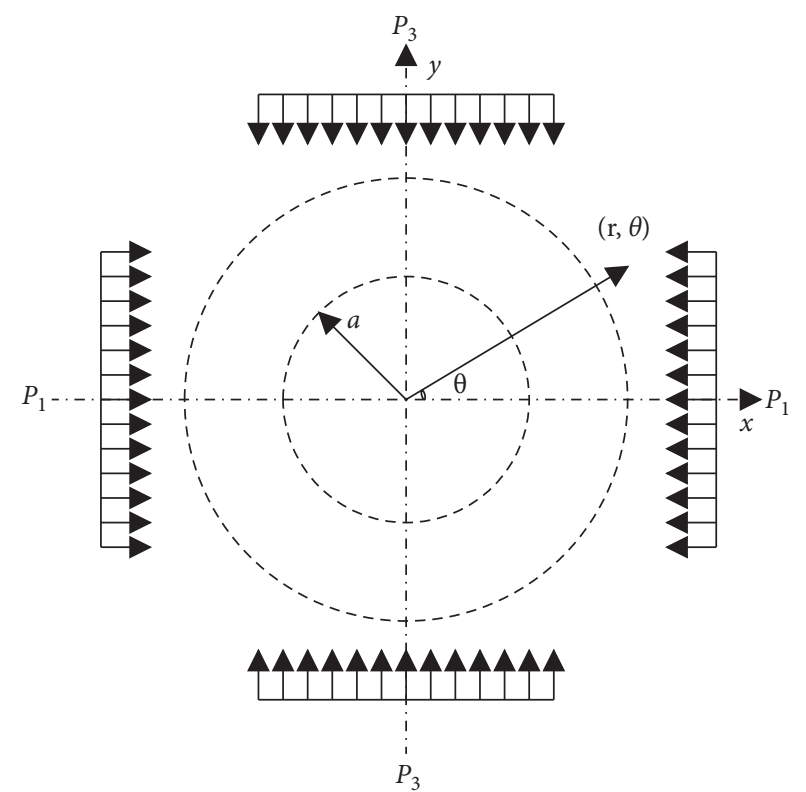

FIGURE 3: Theoretical calculation of butterfly-shaped plastic zone. $P_{1}$ and $P_{3}$ are the maximum and minimum principal stress in surrounding rock stress field; $a$ is the radius of circular roadway; $R$, $\theta$ is the polar coordinates of any location.

motion increment of the confined bulk calculated by using the motion mutation potential function. Moreover, it is also believed [23] that the hypothesis of butterfly coal and gas outburst takes into account the uneven distribution of the ground stress caused by the excavation of the roadways in the working face and gives the stress field state and plastic zone boundary by using the implicit equation of the plastic zone boundary of circular roadway surrounding rock in nonisobaric stress field, which solves the quantitative interpretation of the risk indicators of coal and gas outburst risk indicators. However, the hypothesis is similar to the outburst mechanism hypothesis of pressure-bearing loose mass that the roadway is assumed to be in a plane stress state, which leaves a big difference from the complex stress environments of coal and gas outbursts in practice. Also, the conjecture does not consider the role of physical and mechanical properties of gas and coal in the whole outburst 
process and how these three interact with each other to induce the coal and gas outbursts, thus leaving certain limitations.

Therefore, in spite of the full demonstration of the effects of in situ stress, gas, and tectonic movements by the above theories, researches on the action mechanism of the coal strength on coal and gas outbursts are rare due to the limited testing means and conditions. Strength is one of the important parameters of the physical and mechanical properties of the coal mass, also an essential index to predict the risk of coal and gas outbursts, and the strength value greatly affects the degree of damage of coal and gas outburst accidents [69].

\section{Influencing Factors of Coal Strength}

Differentiation of the coal strength is attributed to different occurrences under various geological conditions against the backdrop of the complicated geologic environment in China. It has been proved that the coal strength is affected by geological structure movement, gas pressure, size effect, and mining disturbance.

(1) Influence of tectonic movement on coal strength The formation and evolution of structures are closely related to coal and gas outbursts, which change the bedding, joints, faults, fractures, and folds in the coal mass, as well as the microscopic pores inside the coal mass. Besides, the changes of the stress environment state of the coal mass have a certain influence on the strength of the coal mass [46]. Han et al. [70] found that the tectonic coal mass is relatively developed in areas where tectonic stress is concentrated, such as compressive fault zones and fold shafts caused by tectonic movements. Also, tectonic movements cause compaction or loosening of micropore structures in the coal mass, and the multi-stage evolution of tectonic stress fields makes most faults go through multi-stage activities, resulting in reduction of the coal strength. Moreover, Cao et al. [71] conducted uniaxial mechanical parameters experiments on coal samples from different areas and different coal ranks, and they also found that the tectonic movements change the tectonic stress fields combined with the statistics of previous uniaxial experiment data. Different tectonic stress fields affect the change speed of coal elastic modulus with uniaxial compression strength, as well as the interaction between the tectonic stress and the coal ranks causes the relationship between the elastic modulus and Poisson's ratio to change, which causes the change of the coal strength in turn. In addition, the tectonic movements have changed the microscopic pore structures inside the coal mass, leaving a significant increase in density and unstable direction of the fractures which causes the change of the coal strength [72].

(2) Influence of gas pressure on coal strength
The gas mainly exists in the micropores and microcracks in coal mass in the forms of adsorption and free state. And the existence of gas greatly causes the change of internal structures of coal mass, which further leads to the change of the coal strength in turn. Han et al. [73] believed that more than $90 \%$ of the coalbed methane is adsorbed on the coal base blocks, which reduces the permeability of coal mass. At the same time, the existence of adsorbed gas reduces the gas migration channels in the coal mass, which is extremely easy to cause the coal mass to fracture and reduce its strength. Similarly, Zhang [74] found that the content of adsorbed gas in coal mass increases with the increase of the gas pressure. Besides, the expansion and deformation of coal mass and the increase of gas pressure reduces the size of structural cohesion and effective stress between coal mass, resulting in the decrease of the coal strength. Generally, the coal structures will become more compact under the condition of gas desorption, with the coal strength increasing to some extent.

Other scholars believe that the existence of gas accelerates the derivation and expansion of internal cracks in coal mass, resulting in the change of the coal strength. $\mathrm{Xu}$ et al. [75] conducted a series of meso-pressure shear experiments on the gas-bearing coal mass and found that the coal cracks continue to expand in the process of experiments, which provides many channels for gas to infiltrate into coal mass. With the continuous development of pressure and shear experiments, the observation surfaces of the coal mass gradually break and fall off. Besides, the broken areas continue to increase, and more likely new cracks evolve, which eventually leads to the penetration of the cracks and the reduction of coal strength. On the basis of previous studies, Bai et al. [76] found that the defect structures such as microcracks and micropores in the coal mass further expand and fracture after the coal mass absorbs gas. Meanwhile, part of the elastic potential energy is transformed into solid surface energy during the fracture process of the defect structures. When the increasing rate of the solid surface energy is equal to the release rate of the elastic energy, the defect structures of the coal mass are in the critical state of fractures.

As a result, the coal strength decreases with the increase in the number of internal cracks, pores, and other defects, which leads to the increase of gas content in coal mass. Conversely, the increase of gas content will accelerate the derivation and expansion of internal cracks, pores, and other defects, in coal mass, which leads to the decrease of coal strength.

(3) Influence of size effect on coal strength

Physical similarity experiments and numerical simulation methods have become the important means to study the mechanism of coal and gas 
outbursts. The research shows that the selection of engineering material specifications affects a series of strength indicators such as compressive strength, tensile strength, and flexural strength, and the size of the material also affects the final results of physical and numerical simulation experiments [77]. Deng [78] took the size effect as the starting point for the problem and found that there is a positive correlation between the coal strength value and size, which is in line with the theoretical function relationship of the Weibull strength size effect. And they also found that there are large number of pores and cracks of different sizes in the coal mass with the increase of the test size of coal mass.

The theoretical relationship between coal strength and size is as follows:

$$
\sigma=\frac{\sigma_{0}}{\left(V / V_{0}\right)^{1 / m}} \Gamma\left(\frac{1}{m+1}\right)
$$

where $\sigma$ is the strength of the coal sample; $\sigma_{0}$ is the strength value of reference sample; $m$ is the test material homogeneity; $V$ is the test sample volume; and $V_{0}$ is the reference sample volume, the laboratory standard sample is usually used as the reference sample.

Zhao et al. [77] believed that the size effect exhibits an obvious sensitivity to the coal strength with the increase of gas pressure when the coal is in the same homogeneous conditions, and the gas pressure has a significant impact on the size effect of coal compressive strength of coal, and heterogeneity of the coal controls the size effect of the coal. Herewith, the author thinks that the influence of size effect on coal strength is mainly manifested in the change of the defect structures such as micropores and microcracks inside the coal mass. In this stage, the increase of the tested coal size increases the possibility of the increase in the number of defect structures inside the coal mass, and this promotes the further initiation and evolution of new cracks due to the coupling effect between gas pressure and stress field, softening the coal further reducing the coal strength.

(4) Influence of mining disturbance on coal strength The underground mining activities in coal mines make the stress redistribute in front of the mining face or heading working face and form the pressure relief zone, the concentrated stress zone, and the stress zone of the original rock with the continuous advancement of the working face [79]. Besides, the vibration of blasting, tunneling machine, shearer cutting, and other mining works will cause the change of internal microcracks of the surrounding coal and rock mass and then cause the change of the coal strength. Wang et al. [80] analyze the stress distribution of the two sides of the roadway in the mining working face by using the numerical simulation method. Also, a regularly concentrated distribution area of stress on the two sides of the roadway in the mining face and the tunneling face is formed with the advance of the working face without considering the tectonic movements. Furthermore, the periodic weighting of the roof in the roadway causes periodic impact damage to the surrounding coal and rock mass, which further causes looseness and damage to the coal and rock. Ultimately, the overall strength of the coal mass is reduced. Li et al. [81] carried out many experiments and found that the vibration leads to the expansion and development of microcracks in coal mass, which gradually forms interpenetrated fissures between the cracks in coal mass and makes the coal strength reduced. They also found the vibration will change the stress state and the occurrence state of gas in coal mass, which increases the possibility of coal and gas outburst due to the rapid increase of the gas pressure.

In addition, the coal strength reduces by using the oftener water injection, hydraulic fracturing and other methods to solve the problems caused by the mining of harder coal. Also, gas extraction activities have a certain impact on the coal strength, which will affect the process of coal and gas outburst $[82,83]$. To sum up, the change of the coal strength affects the distribution state of other factors, such as gas pressure and in situ stress, and it is of great significance to study the influence mechanism of coal strength on outburst prevention and control of coal and gas outburst.

\section{Research Progress of Influence Mechanism of Coal Strength on Outburst}

At present, theoretical achievements have been made on the disaster-causing mechanism of the in situ stress and gas pressure to the coal and gas outbursts in China, and the control effects of coal strength on the occurrence of coal and gas outbursts have been proved. Yuan et al. [84] found a negative correlation between the coal strength and coal and gas outburst. Also, they regard the coal mass as a prominent barrier, and it is more likely to cause coal and gas outburst with the lower coal strength. But there will still be gas outburst accidents under certain conditions with the higher coal strength, and it is lower about the outburst strength than that with the lower coal strength. In addition, some scholars have reached the opposite conclusions due to different experimental conditions. Yuan and Peng et al. $[14,15]$ found that the coal strength increases with the decrease of coal particle size by using their self-designed device of coal and gas outburst. In the outburst process, the quality of outburst coal increases with the increase of coal strength, and there is a linear positive correlation between the relative outburst strength and the coal strength. Furthermore, Zhang et al. [85] carried out a series of simulation experiments for the instant exposure of gas-bearing coals with different strengths and found that the indexes of outburst strength, such as the quality of the outburst coal 
power, distance, and hole depth, decrease with the increase of the coal strength under the same in situ stress and gas pressure. Also, there is a linear negative correlation between outburst indexes and the coal strength, which is manifested as the hindrance of the coal strength against the occurrence of the outburst accidents. During the four stages of inoculation, occurrence, development, and termination of coal and gas outburst, the existence of gas pressure leads to the decrease of the effective stress, which accelerates the failure process of coal mass. Besides, the release of gas internal energy in the failure process of coal mass and its proportion in the total energy increase with the increase of the coal strength, and there is a linear positive correlation between gas internal energy, which becomes the main energy source affecting the occurrence of outburst accidents.

At present, the research on the mechanism of coal and gas outburst induced by the coal strength is relatively insufficient, and the definitive evaluation standard of the outburst strength is not unique. So that it is worth further discussing whether the definitive evaluation standard can be formed only by the single index, such as outburst coal quality, distance or hole depth. To sum up, in the action hypothesis on the four stages of incubation, start-up, development, and stop of coal and gas outburst in China, the effects of in situ stress, gas pressure, and the physical and mechanical properties of coal mass are taken into account comprehensively. Moreover, the control effects of the ground stress and gas pressure in the structural coal seams are believed to mainly lead to the occurrence of the coal and gas outburst. The above studies have indicated that the mechanical properties of coal mass are changed by the geological structure movements, which leads to the occurrence of outbursts, but it is focusing on the distribution laws of in situ stress and gas pressure in the coal seams about outbursts. Besides, the coal strength is one of the important physical mechanical parameters of coal mass, and how to further study coal and gas outburst induced by coal strength is of great significance to the improvement of outburst theories.

\section{Discussion and Outlooks}

By summarizing the mechanism of coal and gas outbursts in China, it can be seen that the existing research mainly focuses on influence of in situ stress, tectonic movement, and gas pressure on the coal and gas outburst. The coal strength is an important physical mechanical property of the coal mass and is closely related with the strength of the outburst. However, research on the effects of the coal strength on the disaster-causing mechanism of the coal and gas outburst is rarely reported. Therefore, further thinking should be given to the following aspect.

(1) The influence mechanism of the coal strength to the coal and gas outburst requires further research. More and more scholars regard in situ stress, gas, physical and mechanical properties, and other influencing factors as an organic whole, and they also comprehensively consider the processes of coal and gas outbursts by constructing different mathematical models, such as spherical shell instability model $[25,26]$ and butterfly coal and gas outburst conjecture $[64,65]$. However, previous studies often set certain preconditions, for example, the hypothesis of spherical shell instability assumes that the coal seam is usually infinite with the isotropic original stress, and the stress concentration caused by mining work should be ignored. Also, the conjecture of butterfly coal and gas outburst simplifies the surrounding rock of the driving roadway into a plane, and the implicit equation of plastic zone boundary of surrounding rock in nonequal pressure stress field is calculated by means of elastic mechanics. By this conjecture, we can solve outburst problems like "pear-shaped cavity," but the scientific issues related to coal and gas outburst, such as gas pressure and coal strength are ignored. How to further determine the critical conditions of the change of the coal strength in the four stages of incubation, occurrence, development, and stop of the outburst and how to quantitatively analyze the influence of coal strength on outburst are particularly important.

(2) Coal and gas outburst includes four stages of incubation, occurrence, development, and stop, in which the coal mass is destroyed gradually under the coupling action of stress field, gas pressure field, vibration field, seepage field, fracture field, and electromagnetic field, and the gas pressure plays the most important role in the process of coal and gas outburst, following by coal strength and in situ stress [84]. Moreover, the tectonic movement has an extremely important impact on the coal strength and induces the coal and gas outburst [42-46]. But the researches only focus on the crushing effect of gas pressure and external stress on coal mass, ignoring the effect of in situ stress and tectonic movement on the coal mass' crushing and stripping. In addition, the in situ stress and tectonic movement can change the opening and closing states of the internal fractures of coal mass and change the gas migration at the same time. Generally, when the coal strength is large enough or the integrity of coal mass is good enough, the gas pressure is insufficient to strip and break the high-strength coal mass, and the coal mass plays an important role in preventing coal and gas outbursts, which affects the test results of coal and gas outbursts. Otherwise, some scholars consider the influence of coal strength on coal and gas outburst from another perspective $[14,15]$. The particle size of the coal size changes the internal porosity of the coal mass, the smaller the particle size, the smaller the porosity, and the better the compactness of the coal mass. The test has indicated that the smaller the particle size of the coal, the better the adsorption feature of the coal, and the higher the temperature of 
coal rises. Under effects of the temperature field, energy stored in the high-strength coal is relatively high; therefore the outburst is instantaneous with a high intensity. The current research focuses on the changing internal energy of the gas stored in the coal due to the changes of the adsorption feature of the gas. Enhancement of the adsorption feature of the gas rises the temperature within the coal mass, thus transporting energy to expand the gas continuously. As a result, the intensity of the outburst may be increased. Under the same gas pressure and ground stress, the coal strength is the main factor that affects the failure form of the coal mass at each stage of the outburst, which is the main influencing factor that controls the storage and release of the elastic energy and gas internal energy. At the same time, the author thinks that how to establish more suitable experiment conditions for underground outburst process and fully consider the coupling effect of multiple fields will be more conducive to study the influence mechanism of physical and mechanical parameters on coal and gas outburst and improve the disaster mechanism of coal and gas outburst.

(3) Research methods of the disaster mechanism of coal and gas outburst need to be further improved in the future. In recent years, the mechanisms of coal and gas outburst have been studied by using rheological mechanics, elastic mechanics, chaos theory, plastic mechanics, rock mechanics, and gas-solid coupling theory, and by means of laboratory similar simulation experiments and numerical simulation methods, but no methods or theories are perfect. In short, they can explain specific coal and gas outburst accidents to a limited extent.

The size effects have greatly changed the coal strength value $[77,78,86]$, and when for the similarity simulation experiments on coal and gas outburst, it is necessary to ensure that the physical and mechanical properties, geometric similarity, movement trend similarity and dynamic similarity of the simulated coal seams according to the similarity theory. But it is difficult to ensure that the final simulation results are consistent with the actual outburst process and outburst characteristics of the mine [87]. Therefore, the physical and mechanical properties of similar simulation materials and raw coal should be the same as possible, avoiding the influence of the size effects on the coal and gas processes caused by the coal strength, and ensuring the rationality of the design of the similar simulation experiments to achieve the quantification and accuracy of experiment on coal and gas outburst. However, the existing devices of the similar simulation experiment limit the accuracy of the experiment results of coal and gas outburst to a certain extent. For example, some of the existing devices [88] are designed with a small size, which is difficult to avoid the influence of boundary effect and hard to simulate the existence of the geological structure of the coal seam and the realization of engineering excavation function. In addition, the sensors can only be placed in the model, which limits the data collection. Or some of the existing devices are designed with a large size, which increases the difficulty of device manufacturing and the periodicity of experiments. Or some of the existing devices are designed with a smaller volume of the gas cavity, which makes it difficult to maintain the adequacy of gas during the testing. Or some of the existing devices have poor airtightness and cannot provide the true triaxial stress loading which is more consistent with the actual engineering difficultly.

In view of the existing problems to the research methods and devices, the existing materials for the similarity simulation test should be improved to conform to the physical and mechanical properties of coal mass. The key factors of coal and gas outburst, such as in situ stress, gas stress, and physical and mechanical properties, should be comprehensively considered, and the scientific and reasonable size (avoiding boundary effects and size effects) should be designed to truly restore the geological structures of the coal seams and gas migration laws and truly restore the stress environment and excavation conditions of the coal seams. At the same time, the device of the similarity simulation test in terms of the four stages of preparation, excitation, development, and stopping (acquiring the critical conditions of each stage) can be obtained from multiple directions. Besides, the priority should be placed on the impact of changes of the macro-mechanical properties of coal mass on its microstructures, and how to use numerical simulation technology more reasonably by means of the computer numerical simulation technology.

\section{Conclusions}

Coal and gas outburst is a complex kinetic process. The current researches have revealed the disaster-causing mechanism of the coal strength to the coal and gas outburst, providing an idea for deep mining as well as prevention and control of the coal and gas outburst.

(1) The research progresses and new understandings of the coal and gas outburst mechanism under the control of different factors are systematically summarized, providing an idea for the future deep mining as well as prevention and control of the coal and gas outburst in China.

(2) Stages of incubation, occurrence, development, and stop of the coal and gas outburst are affected by the coupling effects of the multi-physical field such as in situ stress field, gas pressure field, fissure field, electromagnetic field, and seepage field. The coal strength is the main factor that affects the occurrence of outburst under the same gas pressure and ground stress.

(3) Scientific methods are put forward to improve the research methods of the mechanism of coal and gas outburst. It is imperative to design a scientific size (boundary effects and size effects should be avoided), restore the real coupling conditions of multiple 
geological fields, obtain the critical conditions of each stage from multiple directions, use numerical simulation technology comprehensively, and consider the influence of the changing mechanical properties of macrocoal on its microstructure.

\section{Conflicts of Interest}

The authors guarantee that they have no conflicts of interest to other research studies in this paper.

\section{Acknowledgments}

This work was financially supported by the Natural Science Foundation of Beijing Municipality (Grant no. 8192036), the National Key Research and Development Program of China (Grant no. 2018YFC0808301), the State Key Laboratory Cultivation Base for Gas Geology and Gas Control (Henan Polytechnic University)(Grant no. WS2018B04), the Municipal Research Institute Reform and Development Project (Grant No. BJAST-RD-BMILP202109-03), and the Municipal Research Institute Reform and Development Project (Grant No. BJAST-RD-BMILP202113).

\section{References}

[1] Y. Song, Q. Zou, E. Su, Y. Zhang, and Y. Sun, "Changes in the microstructure of low-rank coal after supercritical $\mathrm{CO}_{2}$ and water treatment," Fuel, vol. 279, no. 3, Article ID 118493, 2020.

[2] Q. Zou, H. Liu, Y. Zhang, Q. Li, J. Fu, and Q. Hu, "Rationality evaluation of production deployment of outburst-prone coal mines: a case study of nantong coal mine in Chongqing, China," Safety Science, vol. 122, Article ID 104515, 2020.

[3] H. Wang, Z. Cheng, Q. Zou et al., "Elimination of coal and gas outburst risk of an outburst-prone coal seam using controllable liquid $\mathrm{CO}_{2}$ phase transition fracturing," Fuel, vol. 284, Article ID 119091, 2021.

[4] M. Gao, S. Zhang, J. Li, and H. Wang, "The dynamic failure mechanism of coal and gas outbursts and response mechanism of support structure," Thermal Science, vol. 23, p. 122, 2019.

[5] Y.-k. Ma, B.-s. Nie, X.-q. He, X.-c. Li, J.-q. Meng, and D.-z. Song, "Mechanism investigation on coal and gas outburst: an overview," International Journal of Minerals, Metallurgy and Materials, vol. 27, no. 7, pp. 872-887, 2020.

[6] W. Gong and D. Guo, "Control of the tectonic stress field on coal and gas outburst," Applied Ecology and Environmental Research, vol. 16, no. 6, pp. 7413-7433, 2018.

[7] W. P. Diamond and S. J. Schatzel, "Measuring the gas content of coal: a review," International Journal of Coal Geology, vol. 35, no. 1-4, pp. 311-331, 1998.

[8] I. W. Farmer and F. D. Pooley, "A hypothesis to explain the occurrence of outbursts in coal, based on a study of west wales outburst coal," International Journal of Rock Mechanics and Mining Sciences \& Geomechanics Abstracts, vol. 4, no. 2, pp. 189-193, 1967.

[9] C. Dong, W. E. Yuan, and L. Nan, "Study on the microwave effect on the physical and mechanical properties of coal," International Journal of Oil Gas and Coal Technology, vol. 18, no. 1-2, 2018.

[10] Z. Sun, L. Li, F. Wang, and G. Zhou, "Desorption characterization of soft and hard coal and its influence on outburst prediction index," Energy Sources Part A Recovery Utilization and Environmental Effects, vol. 2, pp. 1-15, 2019.

[11] L. Wang, Z. Lu, D. P. Chen, Q. Q. Liu, and Z. J. Wen, "Safe strategy for coal and gas outburst prevention in deep-andthick coal seams using a soft rock protective layer mining," Safety Science, vol. 129, Article ID 104800, 2020.

[12] H. B. Zhao and G. Z. Yin, "Study on impact of coal and gas outburst strength caused by principal stress," Disaster Advances, vol. 3, no. 4, pp. 388-391, 2010.

[13] C. Fan, S. Li, D. Elsworth, and Z. Yang, "Experimental investigation on dynamic strength and energy dissipation characteristics of gas outburst-prone coal," Energy Science and Engineering, vol. 8, no. 1-4, 2019.

[14] R. F. Yuan and H. M. Li, Simulation Test and Analysis on CoalGas Outburst at Condition of Different Coal Strength, Commemorating the 50th Anniversary of the Founding of China Coal Society, Editorial Department of Shandong Coal Science and Technology, China Coal Society, Beijing, China, 2012.

[15] S. J. Peng, X. W. Song, J. Xu et al., "Experiment of factors affected to coal and gas outburst intensity based on hypothesis of comprehensive effect," Coal Science and Technology, vol. 44, no. 12, pp. 81-84, 2016.

[16] Q. X. Yu, Mine Gas Prevention and Control, China University of Mining and Technology Press, Xuzhou, China, 1992.

[17] Y. G. Wang, J. P. Wei, and H. T. Sun, "Mechanism analysis of coal or rock dynamic disaster and its prevention and control," Mining Safety \& Environmental Protection, vol. 37, no. 2, pp. 17-19, 2010.

[18] L. J. Wei, S. Li, Z. K. Wei, and M. W. Wang, "Research status and prospects of the influence law of coal and gas outburst on ventilation system," Coal Science and Technology, pp. 1-7, 2021.

[19] F. T. Miao, D. L. Sun, and Q. T. Hu, "The formation mechanism of shock waves in the coal and gas outburst process," Journal of China Coal Society, vol. 38, no. 3, pp. 367-372, 2013.

[20] K. Wang, L. Wang, and F. Du, "Influence of coal powder particle sizes on dynamic characteristics of coal and gas outburst," Journal of China Coal Society, vol. 44, no. 5, pp. 1369-1377, 2019.

[21] A. A. Haryoff, Mine Ventilation, Northeast Institute of technology, Guwahati, India, 1956.

[22] J. Han, H. Zhang, and B. J. Huo, "Discussion of coal and gas outburst mechanism of syncline," Journal of China Coal Society, vol. 8, pp. 908-913, 2008.

[23] Q. F. Wang, Study on the Stress Evolution Process of Coal and Gas Outburst Mechanism in Excavation, China University of Mining and Technology, Beijing, China, 2018.

[24] B. Y. Zhang, Y. Yu, X. Gao, Q. Wu, and Q. Zhang, "Stressstrain characteristics of coal mine gas hydrate-coal mixture under confining pressure unloading," Journal of China Coal Society, pp. 1-12, 2020.

[25] C. L. Jiang and Q. X. Yu, "The hypothesis of spherical shell destabilization of coal and gas outburst," Safety in Coal Mines, vol. 2, pp. 17-25, 1995.

[26] C. L. Jiang, "Study of the reasons for the delay of the coal and gas outburst," China Safety Science Journal, vol. 4, no. 4, pp. 28-32, 1994.

[27] C. L. Jiang, "Forecast model and indexes of coal and gas outburst," Journal of China University of Mining and Technology, vol. 4, pp. 3-5, 1998.

[28] S. N. Zhou and X. Q. He, "Rheological hypothesis of coal and methane outburst mechanism," Journal of China University of Mining and Technology, vol. 19, no. 2, pp. 1-8, 1990. 
[29] X. Q. He and S. N. Zhou, "Rheological hypothesis of coal and methane outburst mechanism," Safety in Coal Mines, vol. 10, 1991.

[30] P. F. Li, "Study on the hypothesis of coal and gas outburst mechanism: two phase fluid hypothesis," Safety in Coal Mines, vol. 11, 1989.

[31] M. Zhang, Z. Xu, and Y. Pan, "A united instability theory on coal (rock) burst and outburst," Journal of China Coal Society, vol. 4, 1991.

[32] B. Liang, Study on the Theory of Solid Fluid Coupling Instability of Coal and Gas Outburst, Northeastern University, Boston, MA, USA, 1994.

[33] B. Liang, M. T. Zhang, Y. S. Pan, and L. G. Wang, "Theory of instability of flow fixation coupling for coal and gas outburst," Journal of China Coal Society, vol. 20, no. 5, pp. 492-496, 1995.

[34] S. L. Lv and J. S. He, "Key layer and stress dike mechanism of coal and gas outbursts," Journal of Chongqing University, vol. 22, no. 6, pp. 80-84, 1999.

[35] J. S. He and S. L. Lv, Geophysical Study on Gas Outburst, China Coal Industry Publishing House, Beijing, China, 1999.

[36] X. Yuan, Study on Mechanism of Coal and Gas Outburst Based on Gas-Driven, Chongqing University, Chongqing, China, 2017.

[37] J. Chen, X. K. Pan, D. Y. Jiang, X. Yuan, X. Jiang, and J. Y. Fan, "Development and application of coal and gas outburst test system based on gas driven," Journal of China Coal Society, vol. 43, no. S2, pp. 460-468, 2018.

[38] Y. P. Cheng, X. L. Zhang, and L. Wang, "Controlling effect of ground stress on gas pressure and outburst disaster," Journal of Mining \& Safety Engineering, vol. 30, no. 3, pp. 408-414, 2013.

[39] H. J. He and X. S. Chen, "Research state and its development trends for control function of geological structure to coal and gas outburst," Journal of Henan Polytechnic University (Natural Science), vol. 28, no. 1, pp. 1-7, 2009.

[40] Z. M. Zhang and Y. G. Zhang, "Three grades of gas-geological maps and their application to gas controlling," Journal of China Coal Society, vol. 4, pp. 455-458, 2005.

[41] M. J. Liu, X. L. Liu, and J. He, "Research on fractal prediction of coal and gas outburst," Journal of China Coal Society, vol. 6 , pp. 3-5, 1998.

[42] J. Han and H. W. Zhang, "The controlling of tectonic evolution to coal and gas outburst," Journal of China Coal Society, vol. 35, no. 7, pp. 1125-1130, 2010.

[43] J. W. Yan, X. B. Zhang, and Z. M. Zhang, "Research on geological control mechanism of coal-gas outburst," Journal of China Coal Society, vol. 38, no. 7, pp. 1174-1178, 2013.

[44] X. S. Zhu and F. Y. Xu, "The controlling effect of tectonic stress field and its evolution on coal and gas outburst," Journal of China Coal Society, vol. 19, no. 3, pp. 304-313, 1994.

[45] L. Shu, K. Wang, Q. G. Qi, S. Fan, L. Zhang, and X. Fan, "Key structural body theory of coal and gas outburst," Chinese Journal of Rock Mechanics and Engineering, vol. 36, no. 3, pp. 347-356, 2017.

[46] F. C. Hao, M. J. Liu, J. P. Wei, and Y. Q. Fu, "The controlling role of gravitational slide structure to coal and gas outburst," Journal of China Coal Society, vol. 37, no. 5, pp. 825-829, 2012.

[47] F. J. Zhao, W. Qian, J. G. Deng, and M. J. Liu, "Tectonic control on coal and gas outburst of coal seam in Malingshan gliding structure," Journal of China Coal Society, vol. 38, no. S1, pp. 112-116, 2013.

[48] C. H. Zhang, Z. G. Liu, T. Xu, J. Liu, and F. Cai, "Coal and gas outburst in steep inclined coal seam uncovered by two reverse drifts and technology of outburst elimination by increasing permeability with blasting," Journal of China Coal Society, vol. 35, no. 1, pp. 85-88, 2010.

[49] Q. T. Hu, S. N. Zhou, and X. Q. Zhou, "Mechanical mechanism of coal and gas outburst process," Journal of China Coal Society, vol. 33, no. 12, pp. 1368-1372, 2008.

[50] J. C. Ou, E. Y. Wang, G. Q. Ma et al., "Coal rupture evolution law of coal and gas outburst process," Journal of China Coal Society, vol. 37, no. 6, pp. 978-983, 2012.

[51] D. Liu, J. Xu, G. Z. Yin, W. Z. Wang, Y. Q. Liang, and J. S. Peng, "Development and applation of multifield coupling testing system for dynamic disaster in coal mine," Chinese Journal of Rock Mechanics and Engineering, vol. 32, no. 5, pp. 966-975, 2013.

[52] K. Gao, G. D. Qiao, Z. G. Liu, J. Liu, F. H. Zhu, and S. C. Zhang, "On classification conception of coal and gas outburst mechanism and its application," Journal of Mining \& Safety Engineering, vol. 36, no. 5, pp. 1043-1051, 2019.

[53] J. P. Tang, S. L. Yang, Y. L. Wang, and J. Q. Lv, "Experiment of coal and gas outbursts under ground stress and gas pressure in deep mines," Rock and Soil Mechanics, vol. 35, no. 10, pp. 2769-2774, 2014.

[54] D. L. Sun, Q. T. Hu, and F. T. Miao, "Motion state of coal-gas flow in the process of outburst," Journal of China Coal Society, vol. 37, no. 3, pp. 452-458, 2012.

[55] X. G. Xie, T. Feng, Y. Wang, and S. Y. Huang, "The energy dynamic balance in coal and gas outburst," Journal of China Coal Society, vol. 35, no. 7, pp. 1120-1124, 2010.

[56] Y. Zhao, B. Q. Lin, T. Liu et al., "Mechanism of multifield coupling-induced outburst in mining-disturbed coal seam," Fuel, vol. 272, Article ID 117716, 2020.

[57] J. Zhang, H. Yan, Q. Zhang, B. Li, and S. Zhang, "Disastercausing mechanism of extremely thick igneous rock induced by mining and prevention method by backfill mining," European Journal of Environmental and Civil Engineering, vol. 24, no. 3, pp. 307-320, 2020.

[58] X. C. Li, B. S. Nie, L. K. Wang, L. C. Dai, and Z. Liu, "Analysis on coal and gasoutburstm echanism underm ultifield coupling action," Coal Science and Technology, vol. 39, no. 5, pp. 64-66+69, 2011

[59] X. Q. He, Disaster Dynamics of Gas Bearing Coal and Rock, China University of Mining and Technology Press, Xuzhou, China, 1995.

[60] D. Y. Guo and D. X. Han, "The stick-slip mechanism of coal and gas outburst," Journal of China Coal Society, vol. 6, pp. 598-602, 2003.

[61] Q. X. Qi, Y. W. Shi, and T. Q. Liu, "Mechanism of instability caused by viscous sliding in rock burst," Journal of China Coal Society, vol. 22, no. 2, pp. 144-148, 1997.

[62] J. R. Wang, C. B. Deng, and H. Z. Deng, "Study on the microcosmic mechanism for coal-gas outbrust," Journal of China Coal Society, vol. 2, pp. 131-135, 2008.

[63] Z. F. Ma and Q. X. Yu, "The pilot study on outburst mechanism for compression disseminated values of coal and gas out of control," Journal of China Coal Society, vol. 3, pp. 329-333, 2006.

[64] N. J. Ma, X. D. Zhao, Z. Q. Zhao, X. F. Guo, H. T. Liu, and H. S. Jia, "Conjecture about mechanism of butterfly shape coal and gas outburst in excavation roadway," Journal of Mining Science and Technology, vol. 2, no. 2, pp. 137-149, 2017.

[65] Z. Q. Zhao, N. J. Ma, X. F. Guo, X. D. Zhao, Y. X. Xia, and Z. K. Ma, "Mechanism conjecture of butterfly rock burst in coal seam roadway," Journal of Mining Science and Technology, vol. 41, no. 11, pp. 2689-2697, 2016. 
[66] Z. Q. Zhao, N. J. Ma, H. T. Liu, and X. F. Guo, “A butterfly failure theory of rock mass around roadway and its application prospect," Journal of China University of Mining and Technology, vol. 47, no. 5, pp. 969-978, 2018.

[67] Z. Q. Zhao, Study on Mechanism and Control Method of Deformation and Failure of Surrounding Rock in Lagged Deformation Mining Roadway, China University of Mining and Technology, Beijing, China, 2014.

[68] X. F. Guo, L. F. Guo, N. J. Ma, Z. Q. Zhao, and L. Chen, "Applicability analysis of the roadway butterfly failure theory," Journal of China University of Mining and Technology, vol. 49, no. 4, pp. 646-653, 2020.

[69] X. S. Zhao, Q. T. Hu, Y. H. Zou, and J. N. Kang, “The fast measurement principium and its application of the consistent coefficient of distant coal beyond working face," Journal of China Coal Society, vol. 32, no. 1, pp. 38-41, 2007.

[70] J. Han, H. W. Zhang, Z. M. Zhu, and J. C. Song, "Controlling of tectonic stress field evolution for coal and gas outburst in Fuxin basin," Journal of China Coal Society, vol. 9, pp. 934938, 2007.

[71] M. M. Cao, Y. S. Kang, Z. Deng, B. F. Tian, Q. Zhao, and H. Y. Wang, "Influence of coal rank and tectonic stress intensity on mechanical properties of coal rock," Coal Science and Technology, vol. 47, no. 12, pp. 45-55, 2019.

[72] R. G. Zhang, L. T. Fang, B. Hu, L. Qin, and L. Xiong, “Tectonic coal structure and pore characteristics of Zhuxianzhuang mine," Coal Geology and Exploration, vol. 43, no. 4, pp. 6-10, 2015.

[73] J. M. Han, Y. Wu, and D. Zhao, "Relationship between methane adsorption and strength about coal sample," Coal, vol. 21, no. 1, pp. 12-13, 2012.

[74] J. S. Zhang, "Experimental study on influence of gas pressure on strength of coal body under adsorption condition," Safety in Coal Mines, vol. 51, no. 1, pp. 26-29, 2020.

[75] J. Xu, X. P. Su, L. C. Cheng, L. Wang, J. Liu, and D. Feng, "Evolution characteristics of meso-cracks of gas-filled raw coal under compression-shear stress," Chinese Journal of Rock Mechanics and Engineering, vol. 33, no. 3, pp. 458-467, 2014.

[76] B. Bai, X. C. Li, Y. F. Liu, Z. M. Fang, and W. Wang, "Preliminary theoretical study on impact on coal caused by interactions between $\mathrm{CO}_{2}$ and coal," Rock and Soil Mechanics, vol. 4, pp. 823-826, 2007.

[77] X. Zhao, J. X. Lv, and J. H. Jiang, "Size effect of modulus of rupture for the larch dimension lumber," Journal of Building Materials, vol. 17, no. 4, pp. 734-737, 2014.

[78] Z. G. Deng, "Study on influencing factors of strength size effect based on bump-prone coal," Coal Science and Technology, vol. 47, no. 8, pp. 59-63, 2019.

[79] J. Xu, W. Zhou, D. Liu, S. C. Li, and H. Y. Tan, “Temperature and acoustic emission characteristics of coal in the process of outburst under the influence of mining," Journal of China Coal Society, vol. 38, no. 2, pp. 239-244, 2013.

[80] Z. Wang, Q. T. Hu, G. C. Wen, and D. L. Sun, "Study on the distribution laws of mining pressure field and its control action on dynamic disasters in coal mines," Journal of China Coal Society, vol. 36, no. 4, pp. 623-627, 2011.

[81] X. C. Li, B. S. Nie, and X. Q. He, "Mechanism of coal and gas bursts caused by vibration," Chinese Journal of Engineering, vol. 33, no. 2, pp. 149-152, 2011.

[82] X. Qi and J. J. Geng, "Experimental study on the effect of water injection to coal strength," Shaanxi Coal, vol. 31, no. 5, pp. 44-45, 2012.
[83] Z. Y. Sun, Z. Zhang, and Z. G. Wu, "Field test analysis of gas drainage to coal strength and anchor character," Coal Mining Technology, vol. 22, no. 5, pp. 45-48, 2017.

[84] J. Xu, D. Liu, S. J. Peng, X. Wu, and Q. Lu, "Experimental research on influence of particle diameter on coal and gas outburst," Chinese Journal of Rock Mechanics and Engineering, vol. 29, no. 6, pp. 1231-1237, 2010.

[85] Q. H. Zhang, S. C. Li, H. P. Wang et al., "Influence mechanism and outburst characteristics during revealing different intensity coal containing gas," Journal of Mining \& Safety Engineering, vol. 34, no. 4, pp. 817-824, 2017.

[86] Y. Zhao, X. H. Li, Y. Y. Lu, and Y. Kang, "Influence of gas pressure on compressive strength size effect of inhomogenous coal," Journal of China Coal Society, vol. 34, no. 8, pp. 1081-1085, 2009.

[87] Y. J. Liu, L. Yuan, J. H. Xue, Z. C. Tian, C. R. Duan, and B. L. Chen, "Research status and development trend of mechanism and simulation test of coal and gas outburst," Industry and Mine Automation, vol. 44, no. 2, pp. 43-50, 2018.

[88] Y. Y. Lu, Z. Y. Peng, B. W. Xia, P. Yu, and C. N. Ou, "Multifunctional physical model testing system of deep coal petrography engineering-coal and gas outburst simulation experiment," Journal of China Coal Society, vol. 45, no. S1, pp. 272-283, 2020. 